\title{
Working Principles of Vibroelectric Nano Generator in Wireless Sensor Networks: A Review
}

\author{
Jee Siang, Lim Meng Hee, and Mohd. Salman Leong
}

Institute of Noise and Vibration, Faculty of Mechanical Engineering, Universiti Teknologi Malaysia, Jalan Sultan Yahya Petra, 54100 Kuala Lumpur, Malaysia

\begin{abstract}
Wireless sensor networks are solely dependent on battery limits the operation as well as maintenance effort. Vibroelectric nano generator able to prolong the operation by converting ambient vibration and power the equipment. The working principles includes Piezoelectric, Electromagnetic, Triboelectric, Magnetostrictive and Flexoelectric. This paper discussed the principle operations and key function materials, the advantages and disadvantages are specified. Hybrid incorporated with solar, thermal or radio frequency is considered as current trend of nano generator with further improvement as future work.
\end{abstract}

\section{Introduction}

Generating electricity from the environment has granted substantial attention recently due to advancement wireless sensor networks (WSN), microelectromechanical systems (MEMS) and Internet of Things (IoT) [1]-[4]. Existing WSN is heavily rely on battery or wired connection as energy sources [4], however the main obstacle for batteries are limited energy density, restricted life cycle, inevitable periodic replacement, labour cost, current leakage (even if not in use) as well as hazardous disposal after usage [4], [5].

Therefore, vibration energy which is abundant from ambient is explored to substitute batteries and wired power, subsequent providing a reliable and continuous supply of energy with lower installation and maintenance cost [4], [6], [7]. Vibroelectric nano generator (VENG) is ideal for long-life WSN for structural health monitoring (SHM)[6], [8], in many applications such as aerospace \& deep sea operation, mining field, nuclear reactor sensor and implantable bio-sensing device[5].

\section{Source of Ambient Energy}

Ambient energies were not substantial for utilization due to intermittent characteristic and considerable high wattage electronics before 2000. WSN benefited with recent advancement of nanotechnology where WSN energy usage rated around $8^{\sim} 12 \mathrm{~mW}[4]$, [9]. Fig. 1 shows the potential energy sources available in ambient[7], [10], [11].

Fig.1 Potential energy sources from ambient to power

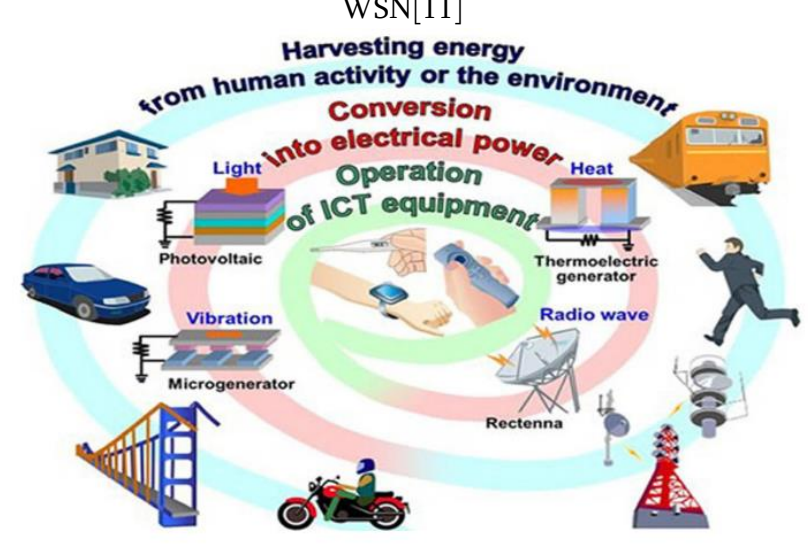

3. Principles

Vibration energy can be captured and converted into electricity for low-power electronics or battery[10]. Among the principles, Piezoelectric (PE) is the most common. Fig.2 shows the variety of working principles for VENG.

Fig.2 The principles of VENG

\footnotetext{
* Corresponding author: yapjeesiang@gmail.com
} 


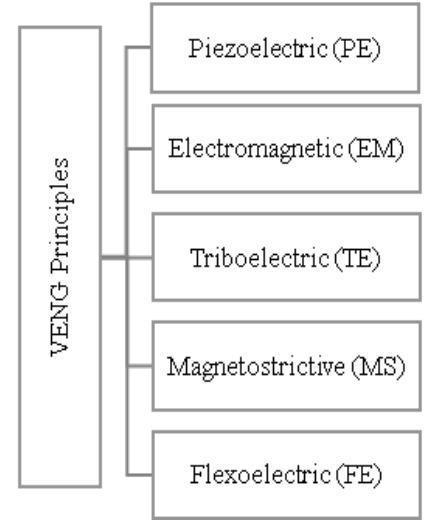

\subsection{Piezoelectric}

Piezoelectric (PE) material produces electric or voltage when the material experienced mechanical stress or strain [5], [12]. The common PE materials are aluminium nitride (AIN), gallium arsenide (GaAs), lead zirconate titanate (PZT), quartz $\left(\mathrm{SiO}_{2}\right)$, polymer polyvinylidene fluoride (PVDF) and recently discovered PE nanogenerators Zinc Nanowires $(\mathrm{ZnO})$ [5], [13]. Fig.3 shows PE effects when experiencing mechanical effort and vice versa[14].

Fig.3 Electromechanical conversion of Piezoelectric Phenomena[14]

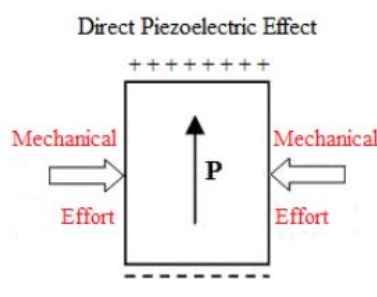

Change Apparition

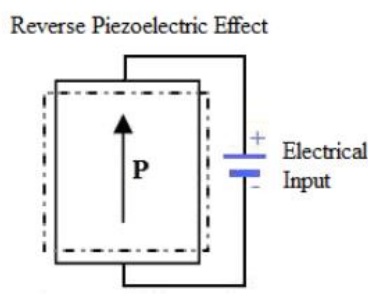

Shape Deformation
The advantages of $\mathrm{PE}$ are simplicity in term of structures, the basic model made of thin layer PE. PE does not greatly affected by external or internal electromagnetic waves [15]. However PE is shortcoming in term of life span especially depolarization, known as electric fatigue after some switching[15], [16]. Ceramic type PE undesirable properties of brittleness and lower coupling coefficient in general. PVDF material show deprived adhesion to electrode materials and low coupling coefficient however comprise of flexible nature [15], [17]. In addition, $\mathrm{PE}$ performance and lifetime deteriorated in high temperature operations[15], [16]. PZT is more desirable by scientists due to relatively high coupling coefficient compared with other PE materials although PZT is brittle [17], [18].

\subsection{Electromagnetic}

Electromagnetic (EM) VENG converts the ambient vibration's mechanical energy to electrical when relative motion occurs between a magnetised body and conductive coil, according to Faraday's law: an electromotive force $(V)$ is generated

$$
\mathrm{V}=\mathrm{N} d \Phi / \mathrm{dt}
$$

where $\mathrm{N}$ is number of turn for the conductive coil, $\Phi$ is the magnetic flux over a single loop[12].

Fig.4 The configuration of simple EM VENG[19]

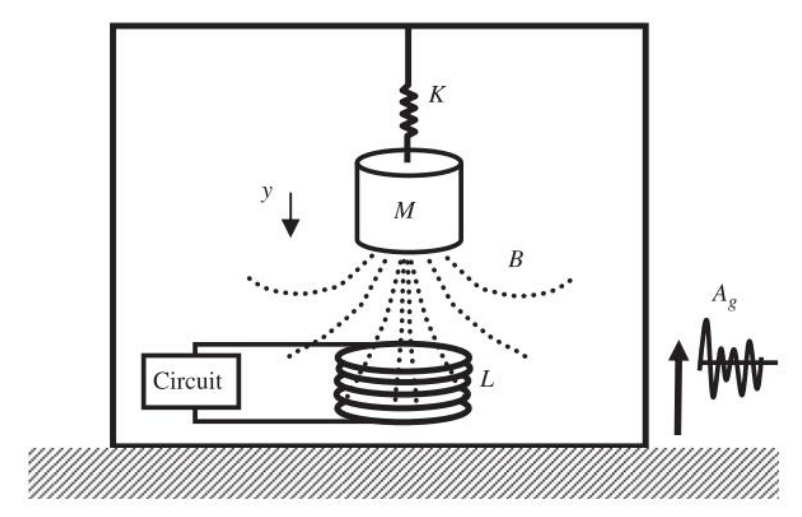

A simple EM configuration is shown in Fig.4, the motional electromotive force (emf) is

$$
\begin{gathered}
e m f=\int d L \cdot(v \times x) \text { or } \\
\left.e m f=-d \int B \cdot d A / d t\right)
\end{gathered}
$$

where $\mathrm{dL}$ is the differential length vector along the inductor coil, $\mathrm{B}$ is the magnetic field vector, and $\mathrm{v}$ is the velocity vector of the magnet. The differential elemental area is dA, so $\Phi=\mathrm{B} \cdot \mathrm{dA}$ [19].

$\mathrm{EM}$ operates in scenario of resonator construction for low frequency conversion, higher conversion efficiency compared to PE VENG [15], [19]. EM required low internal impedance circuit results the generation of high power and current but lower voltage compared $\mathrm{PE}[20]$. While EM performs well in meso-scale, the difficulty to fabricate magnetic coil in micro/nano-scale is a challenge. EM is also vulnerable to electromagnetic waves interference, and 
experiencing damping fiction loss, coil windage loss as well as magnet deterioration[15], [19], [21].

\subsection{Triboelectric}

Triboelectric (TE) generates static electricity with motion between 2 surfaces of a charged capacitor, resulting variation of potential difference [5], [12]. The principle of $\mathrm{TE}$ is illustrated in Fig.5, where electrostatic charges occurred on 2 different material surfaces are contacting each other, the potential of TE charges decrease after vibration energy mechanically separates the 2 surfaces, the potential drop initialize electrons to flow between the electrodes attached each surfaces and connected with a circuit [22].

Fig.5 The four fundamental of TE VENG: (a) Vertical motion, (b) Sliding, (c) single-electrode, and (d) Freestanding [22], [23]

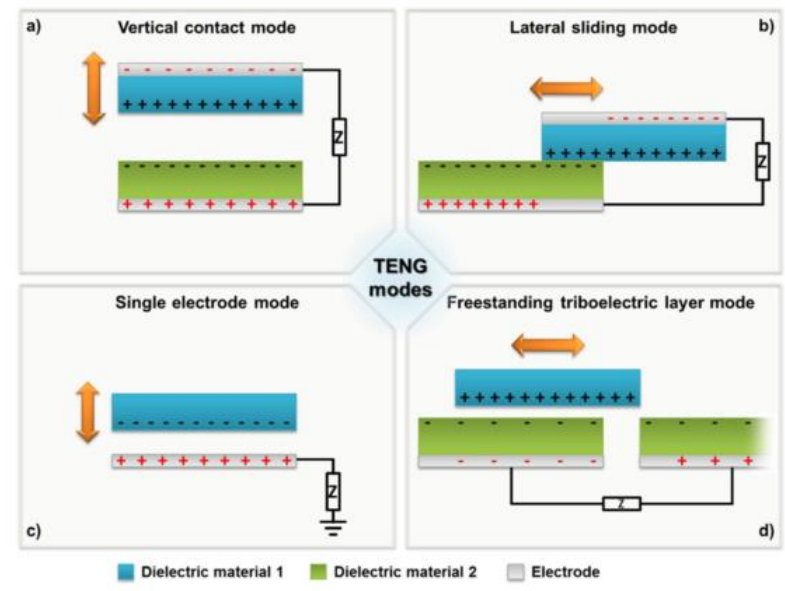

TE VENG able to operates without smart materials unlike PE VENG, thus likely higher system life span. Nevertheless, TE generates relatively small energy density, and external voltage sources is required for VENG operation[15]. The output voltage is tremendously high (usually more than $100 \mathrm{~V}$ ), which is less compatible to MEMS or WSN practically [15].

\subsection{Magnetostrictive}

Magnetostrictive (MS) materials for example irongallium alloy (Galfenol) and Metglas deforms once an altering magnetic field is induced, the deformation sequentially apply stress to PE material for electricity production[12], [24]. MS VENG is grounded on Villari effect principle as shown in Fig.6, where entirely ferromagnetic materials intrinsic potential of MS due to internal electrons motion of the particles, orbital magnetic moment is superpositioned into atomic magnetic moment when the electron spins about its particular axis[25], [26].

Fig.6 (a) Natural magnetism and arbitrary orientation of magnetic moments without external field, (b) the alignment of moment under external magnetic field [26]

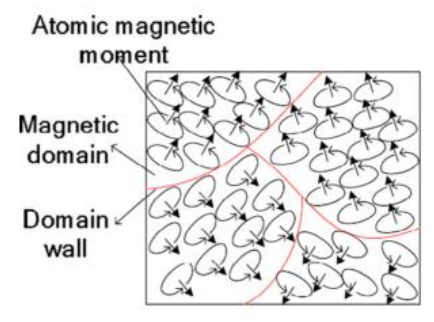

(a)

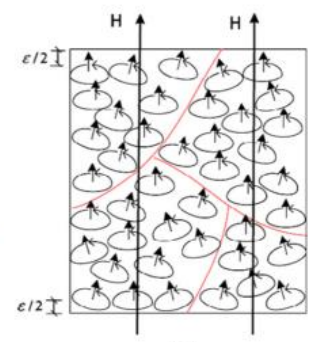

(b)
Fig.7 show the basic MS VENG model, a MS material with 1 length is surrounded by a solenoid with $\mathrm{N}$ turns, assuming periodic forcing $\sigma=\sigma(\mathrm{t})$, the H-field of the current $i$ equals $\mathrm{H}_{i}=\mathrm{Ni} / \mathrm{l}, \mathrm{H}_{0}$ is the biased magnetic field and $\mathrm{R}$ is the circuit load resistance. The power $(\mathrm{P})$ generated equal as following [27]

$$
\begin{aligned}
& \mathrm{P}=\mathrm{R} r^{2} \quad \text { or } \\
& \mathrm{P}=\mathrm{R}^{2}\left(\mathrm{~h}-\mathrm{H}_{0}\right)^{2} / \mathrm{N}^{2}
\end{aligned}
$$

Fig.7 The basic model of MS VENG [27]

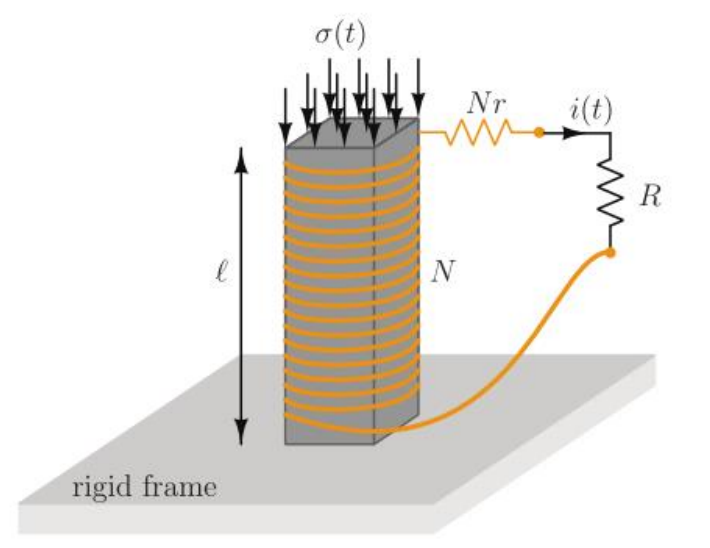

MS VENG generally demonstrate high energy density and extended life cycles [25], MS material induces magnetic field during strain, and electrical current is 
picked up by the coil due to the changing magnetic field according to Faraday's law. Jafari et.al. [28] claimed MS VENG performed better low frequency harvesting compared to PE type [28]. Simulations were conducted on Galfenol rods, magnetic flux density of 1.1 Tesla(T) by a MS VENG was attained sufficient to power VENG for WSN[29].

The advantages of MS over PE are less prone to aging, depolarization, charge leakage and brittleness [24]. MS materials have higher flexibility and able to sustain severer environment but requires external magnetic field during operation[30].

\subsection{Flexoelectric}

Flexoelectric (FE) characteristic exist in all dielectrics materials, where strain gradients allows development polarization even the material is non-piezoelectric[31], [32], shown in Fig.8 a FE materials experiencing polarization when deformed[16].

Fig.8 Polarization due to bending of a centrosymmetric FE beam[16]

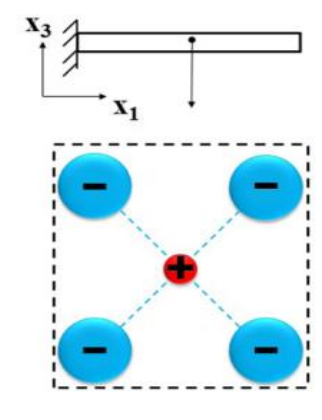

Undeformed

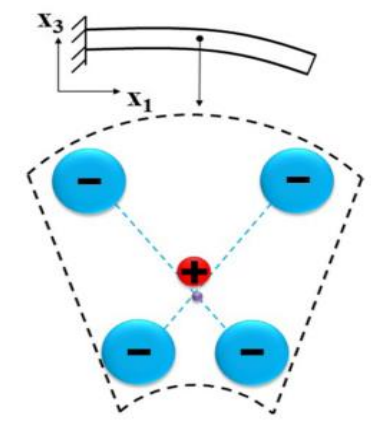

Deformed
FE polymers are able to sustain great strains which ideal for VENG in wearable applications since human/animal movement requires high train/stroke [31], and considerable FE effects in micro/nano-scale conceivably outperforming PE in some situations [16]. However in order for FE VENG to generate an energetic cycle, pseudo-piezoelectric mode is included in the system which required supplementary bias voltage [31]. Other than that, due to limited FE studies, the current FE designs exhibit immature FE coefficient, and unfamiliar degradation property in nano-scale. The common FE material is barium strontium titanate(BST) is ferroelectric with high dielectric permittivity, which generates narrow and sharp dielectric peak during the ferroelectric to paraelectric phase transition temperature, hence inappropriate for wide temperature range applications[33]. Lastly, fabrication of FE VENG system in nanostructures is comparatively complicated[33].

Mahanty et. al.[34] tested a flexible sponge-like nanaogenerator (FSNG) with $\mathrm{ZnO}$ as PE materials and povly-vinylidene fluorideehexafluoropropylene (PVDF-HFP) as FE materials. The FSNG achieves a power density of $1.21 \mathrm{~mW} / \mathrm{cm}^{2}$ and energy conversion efficiency around $0.3 \%$, with $9 \mathrm{~V}$ of open-circuit potential difference, $1.3 \mu \mathrm{A} / \mathrm{cm}^{2}$ of short circuit current, when a mechanical impact of $0.36 \mathrm{MPa}$ stress amplitude is applied above the surface[34].

\section{Comparison}

Each principle take respectively advantages and disadvantages, the relatively comparison in shown in Table I [5], [35]. EM VENG is more applicable in large scale structure such as building and transportation with low vibration frequency $(<20 \mathrm{~Hz})$, while $\mathrm{PE}$ is feasible to micro/meso-scale provided the vibration frequency is high $(>100 \mathrm{~Hz})$. Others VENG methods show potential in micro/nano-scale system such as MEMS and WSN, however the technology is not as mature as EM \& PE.

Table 1. The advantages and disadvantages of corresponding VENG[5], [35]

\begin{tabular}{|c|c|c|}
\hline \multirow{2}{*}{ Principle } & \multicolumn{2}{|c|}{ Properties } \\
\hline & Advantage & Disadvantage \\
\hline $\mathrm{PE}$ & $\begin{array}{l}\text { - Simple structure in } \\
\text { small scale } \\
\text { - High output voltage } \\
(>5 \mathrm{~V}) \\
\text { - High Coupling } \\
\text { Coefficient }\end{array}$ & $\begin{array}{l}\text { - Low output current } \\
\text { - Low strain limit } \\
\text { - Brittle }\end{array}$ \\
\hline EM & $\begin{array}{l}\text { - Simple construction } \\
\text { in large scale } \\
\text { - Low output } \\
\text { Impedance } \\
\text { - Higher output } \\
\text { current }\end{array}$ & $\begin{array}{l}\text { - Low output } \\
\text { voltage }(<1 \mathrm{~V}) \\
\text { - Limitation in } \\
\text { downsizing } \\
\text { - Affected by } \\
\text { electromagnetic } \\
\text { field }\end{array}$ \\
\hline $\mathrm{TE}$ & $\begin{array}{l}\text { - Very high output } \\
\text { voltage }(>100 \mathrm{~V}) \\
\text { - No need of smart } \\
\text { material } \\
\text { - Ease of voltage } \\
\text { rectifying and } \\
\text { frequency tuning }\end{array}$ & $\begin{array}{l}\text { - Bias Voltage is } \\
\text { required } \\
\text { - High impedance } \\
\text { needed } \\
\text { - Low output current }\end{array}$ \\
\hline MS & $\begin{array}{l}\text { - High energy } \\
\text { density } \\
\text { - Long life cycle }\end{array}$ & $\begin{array}{l}\text { - Affected by } \\
\text { Electromagnetic } \\
\text { field } \\
\text { - Limitation in } \\
\text { downsizing }\end{array}$ \\
\hline
\end{tabular}




\begin{tabular}{|c|c|c|}
\hline \multirow{2}{*}{ Principle } & \multicolumn{2}{|c|}{ Properties } \\
\cline { 2 - 3 } & Advantage & Disadvantage \\
\hline \multirow{3}{*}{ FE } & \multirow{3}{*}{ - High strain limit } & - High dielectric \\
& & permittivity \\
& & - Low stress limit \\
\hline
\end{tabular}

\section{Hybrid}

Hybrid VENG utilizes other ambient energy source instead of vibration energy only. The objective is to maintain a continuous energy supply and complement each the intermittent ambient availability [36]. The ambient sources such as sunlight, thermo gradients, and electromagnetic waves were explored to combined in the hybrid VENG[13]. For example, thermoelectric and EM VENG was conducted by Töreyin et. al. [5], using Chromel-Alumel (Cr-Al) as thermoelectric material while Neodymium Iron Boron $(\mathrm{NdFeB})$ magnet as EM components of the cantilever hybrid VENG design[5]. The ambient temperature acted as heat source for the thermoelectric module as shown in Fig.8. The designed prototype size at $9.5 \mathrm{~mm} \times 8 \mathrm{~mm}$ x $6 \mathrm{~mm}$ generated peak voltage of $16.7 \mathrm{mV}$ and power of $1.91 \mathrm{nW}$ with vibration frequency at $3.45 \mathrm{kHz}[5]$.

Fig.9 Schematic diagram of Thermoelectric and EM Hybrid VENG[5]

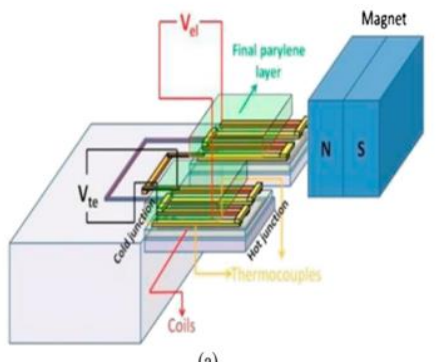

(a)

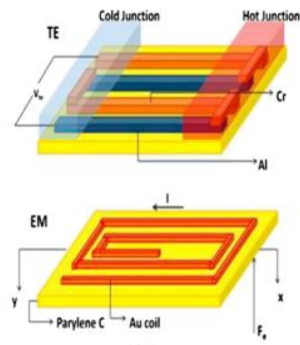

(b)
There are numerous energy sources compactible with vibration energy to form a hybrid system, among are solar radiation, wind flow, acoustic energy, microbial fuel cell and water stream as shown in Fig.10 [7], [20], [37]. H. Li et al. investigated a solar-vibration hybrid VENG which was effective and consistent for continuous energy supply of WSN, the tests were in consideration of 24-hour surrounding fluctuations including rainy and night conditions [7]. As well with Y.He et al, designed a Photovoltaic-PE VENG hybrid, and assisted by Li-ion battery and supercapacitor as energy storage[38].

Fig.10 Potential architecture of multisource energy harvesting system [7]

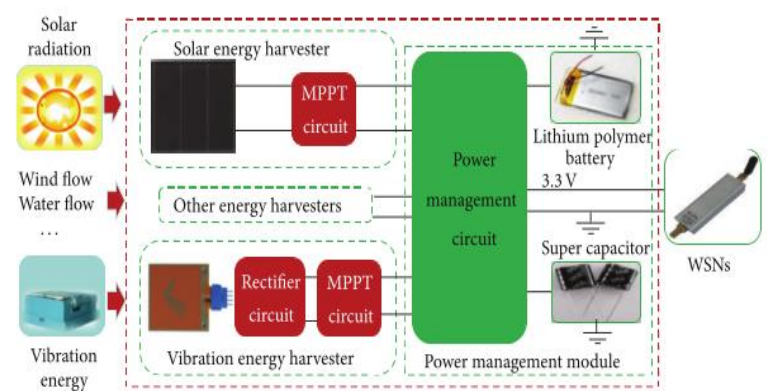

A hybrid VENG consists of 3 input sources of energy namely radio frequency signal, thermal and vibration, capable of delivering $3.3 \mathrm{~V}$ of output voltage, $6.5 \mathrm{~mW}$ of output power and $90 \%$ of efficiency when all input sources are simultaneously harvested[36]. The drawback is inclusion of multiple powered hybrid encounter issue such as system integration, higher fabrication cost and overall volumetric packaging for WSN.

\section{Conclusion}

Vibration energy from ambient is preferable to stretch the mission life of conventional battery for WSN application[11]. The main challenge of VENG is unable to harvest effectively in the stochastics and wide bandwidth of ambient vibration[15]. Reliable and cost effective VENG fabrication methods are required since WSN and MEMS micro scaling are expected to continue, thus micromachining techniques are the gap for commercialization of VENG[39]. Hybrid VENG incorporated with other ambient energy such as thermal, solar and radio frequency can fulfil the vibration-based generator shortage, and future work shall be concentrated in system integration, fabrication cost and packaging.

\section{Acknowledgment}

The authors would like to extend their greatest gratitude to the Institute of Noise and Vibration UTM for funding the study under the Higher Institution Centre of Excellence (HICoE) Grant Scheme (R.K130000.7809.4J225, R.K130000.7809.4J226, )

\section{Reference}

[1] E. Arroyo, A. Badel, F. Formosa, Y. Wu, and J. Qiu, "Comparison of electromagnetic and piezoelectric vibration energy harvesters: Model and experiments," Sensors Actuators, A Phys., vol. 183, pp. 148-156, 2012.

[2] S. S. Balpande, R. S. Pande, and R. M. Patrikar, "Design and low cost fabrication of green vibration energy harvester," Sensors Actuators, A Phys., vol. 251, pp. 134-141, 2016.

[3] K. H. Hui, M. H. Lim, M. S. Leong, and S. M. Al- 
Obaidi, "Dempster-Shafer evidence theory for multibearing faults diagnosis," Eng. Appl. Artif. Intell., vol. 57, pp. 160-170, 2017.

[4] F. K. Shaikh and S. Zeadally, "Energy harvesting in wireless sensor networks: A comprehensive review," Renew. Sustain. Energy Rev., vol. 55, pp. 1041-1054, 2016.

[5] A. R. M. Siddique, S. Mahmud, and B. Van Heyst, "A comprehensive review on vibration based micro power generators using electromagnetic and piezoelectric transducer mechanisms," Energy Convers. Manag., vol. 106, pp. 728-747, 2015.

[6] S. P. Pellegrini, N. Tolou, M. Schenk, and J. L. Herder, "Bistable vibration energy harvesters: A review,” J. Intell. Mater. Syst. Struct., vol. 24, no. 11, pp. 1303-1312, 2012.

[7] H. Li, G. Zhang, R. Ma, and Z. You, "Design and Experimental Evaluation on an Advanced Multisource Energy Harvesting System for Wireless Sensor Nodes," vol. 2014, 2014.

[8] R. Garcia et al., "Piezoelectric energy harvesting: application to data center monitoring," Sens. Rev., vol. 35, no. 4, pp. 401-408, 2015.

[9] K. Yamamoto, T. Fujita, A. Badel, F. Formosa, K. Kanda, and K. Maenaka, "Energy Estimation for Electret Harvester with Nonlinear Spring," Proceedings, vol. 1, no. 4, p. 585, 2017.

[10] Y. Suzuki, "Recent progress in MEMS electret generator for energy harvesting," IEEJ Trans. Electr. Electron. Eng., vol. 6, no. 2, pp. 101-111, 2011.

[11] V. Bhatnagar and P. Owende, "Energy harvesting for assistive and mobile applications," Energy Sci. Eng., p. n/a-n/a, 2015.

[12] S. Naifar, S. Bradai, C. Viehweger, and O. Kanoun, "Survey of electromagnetic and magnetoelectric vibration energy harvesters for low frequency excitation," Meas. J. Int. Meas. Confed., 2015.

[13] C. Xu, C. Pan, Y. Liu, and Z. L. Wang, "Hybrid cells for simultaneously harvesting multi-type energies for self-powered micro/nanosystems," Nano Energy, vol. 1, no. 2, pp. 259-272, 2012.

[14] A. Kokkinopoulos, G. Vokas, and P. Papageorgas, "Energy harvesting implementing embedded piezoelectric generators-The potential for the Attiki Odos traffic grid," Energy Procedia, vol. 50, pp. 1070-1085, 2014.

[15] C. Wei and X. Jing, "A comprehensive review on vibration energy harvesting: Modelling and realization," Renew. Sustain. Energy Rev., vol. 74, no. November 2016, pp. 1-18, 2017.

[16] Q. Deng, M. Kammoun, A. Erturk, and P. Sharma, "Nanoscale flexoelectric energy harvesting," Int. J. Solids Struct., vol. 51, no. 18, pp. 3218-3225, 2014.

[17] S. Anton, K. Farinholt, and A. Erturk, "Piezoelectret foam-based vibration energy harvesting," J. Intell. Mater. Syst. Struct., vol. 25, no. 14, pp. 1681-1692, 2014.

[18] F. Li, Y. You, Y. Lu, and Y. Pan, "Human Centered Computing," vol. 8944, no. 301, pp. 15-25, 2015.

[19] N. G. Elvin and A. A. Elvin, "An experimentally validated electromagnetic energy harvester," J. Sound Vib., vol. 330, no. 10, pp. 2314-2324, 2011.

[20] F. U. Khan and I. Ahmad, "Review of energy harvesters utilizing bridge vibrations," Shock Vib., vol.
2016, 2016

[21] S. Zhu, W. ai Shen, and Y. lin Xu, "Linear electromagnetic devices for vibration damping and energy harvesting: Modeling and testing," Eng. Struct., vol. 34, pp. 198-212, 2012.

[22] Z. L. Wang, "On Maxwell's displacement current for energy and sensors: the origin of nanogenerators," Mater. Today, vol. 00, no. 00, pp. 1-9, 2017.

[23] Q. Zheng, B. Shi, Z. Li, and Z. L. Wang, "Recent Progress on Piezoelectric and Triboelectric Energy Harvesters in Biomedical Systems," Adv. Sci., pp. 123, 2017.

[24] J. Davidson and C. Mo, "Recent Advances in Energy Harvesting Technologies for Structural Health Monitoring Applications," Smart Mater. Res., vol. 2014, p. 14, 2014.

[25] Z.-W. Fang, Y.-W. Zhang, X. Li, H. Ding, and L.-Q. Chen, "Integration of a nonlinear energy sink and a giant magnetostrictive energy harvester," J. Sound Vib., vol. 391, pp. 35-49, 2016.

[26] A. Ghorbanpour Arani and Z. Khoddami Maraghi, “A feedback control system for vibration of magnetostrictive plate subjected to follower force using sinusoidal shear deformation theory," Ain Shams Eng. J., vol. 7, no. 1, pp. 361-369, 2016.

[27] D. Davino, P. Krejčí, A. Pimenov, D. Rachinskii, and C. Visone, "Analysis of an operator-differential model for magnetostrictive energy harvesting," Commun. Nonlinear Sci. Numer. Simul., vol. 39, pp. 504-519, 2016.

[28] H. Jafari, A. Ghodsi, S. Azizi, and M. R. Ghazavi, "Energy harvesting based on Magnetostriction, for low frequency excitations," Energy, vol. 124, pp. 1-8, 2017.

[29] B. Rezaeealam, "Finite element analysis of magnetostrictive vibration energy harvester," COMPEL - Int. J. Comput. Math. Electr. Electron. Eng., vol. 31, no. 6, pp. 1757-1773, Nov. 2012.

[30] M. Ibrahim and A. Salehian, "Modeling, fabrication, and experimental validation of hybrid piezomagnetostrictive and piezomagnetic energy harvesting units," J. Intell. Mater. Syst. Struct., vol. 26, no. 10, p. 1045389X14538540, 2014.

[31] F. Belhora, P. Cottinet, and D. Guyomar, "Hybridization of electrostrictive polymers and electrets for mechanical energy harvesting," Sensors Actuators A ..., vol. 183, pp. 50-56, 2012.

[32] L. Wang, M. Lallart, L. Petit, L. Lebrun, P.-J. Cottinet, and D. Guyomar, "Low-cost charge of electrostrictive polymers for efficient energy harvesting," J. Intell. Mater. Syst. Struct., vol. 26, no. 16, pp. 2123-2136, 2014.

[33] X. Jiang, W. Huang, and S. Zhang, "Flexoelectric nano-generator: Materials, structures and devices," Nano Energy, vol. 2, no. 6, pp. 1079-1092, 2013.

[34] B. Mahanty, S. K. Ghosh, S. Garain, and D. Mandal, "An effective flexible wireless energy harvester/sensor based on porous electret piezoelectric polymer," Mater. Chem. Phys., vol. 186, pp. 327-332, 2017.

[35] L. Vinet and A. Zhedanov, "A 'missing' family of classical orthogonal polynomials," J. Polym. Sci. PART B Polym. Phys., vol. 50, no. 8, pp. 523-535, Nov. 2010. 
[36] T. N. T. Mohamad, J. Sampe, and D. D. Berhanuddin, "Architecture of micro energy harvesting using hybrid input of RF, thermal and vibration for semi-active RFID tag," Eng. J., vol. 21, no. 2, pp. 183-197, 2017.

[37] K. V. Selvan and M. S. Mohamed Ali, "Micro-scale energy harvesting devices: Review of methodological performances in the last decade," Renew. Sustain. Energy Rev., vol. 54, pp. 1035-1047, 2016.

[38] Y. He, Z. You, and X. Wang, "Modelling of a hybrid energy system for autonomous application," Int. $J$. Adv. Robot. Syst., vol. 10, pp. 1-6, 2013.

[39] A. S. M. Zahid Kausar, A. W. Reza, M. U. Saleh, and H. Ramiah, "Energizing wireless sensor networks by energy harvesting systems: Scopes, challenges and approaches," Renew. Sustain. Energy Rev., vol. 38, pp. 973-989, 2014. 